\section{Cardiomyocytes, endothelial cells and cardiac fibroblasts: S100A1's triple action in cardiovascular pathophysiology}

\author{
David Rohde', Martin Busch', Anne Volkert', Julia Ritterhoff', Hugo A Katus ${ }^{1,2}$, Karsten \\ Peppel ${ }^{3,4}$ \& Patrick Most ${ }^{* 1,2,3,4}$
}

\begin{abstract}
Over the past decade, basic and translational research delivered comprehensive evidence for the relevance of the $\mathrm{Ca}^{2+}$-binding protein S100A1 in cardiovascular diseases. Aberrant expression levels of S100A1 surfaced as molecular key defects, driving the pathogenesis of chronic heart failure, arterial and pulmonary hypertension, peripheral artery disease and disturbed myocardial infarction healing. Loss of intracellular S100A1 renders entire $\mathrm{Ca}^{2+}$-controlled networks dysfunctional, thereby leading to cardiomyocyte failure and endothelial dysfunction. Lack of S100A1 release in ischemic myocardium compromises cardiac fibroblast function, entailing impaired damage healing. This review focuses on molecular pathways and signaling cascades regulated by S100A1 in cardiomyocytes, endothelial cells and cardiac fibroblasts in order to provide an overview of our current mechanistic understanding of S100A1's action in cardiovascular pathophysiology.
\end{abstract}

Cardiovascular diseases are projected to remain the number one cause of morbidity and mortality beyond 2030 embodying a growing social and economic burden worldwide [1,2]. Although therapeutic options for heart failure, myocardial infarction, arterial and pulmonary hypertension as well as peripheral artery disease have progressed, cardiovascular disorders yet remain an area with significant unmet medical need due to the mostly symptomatic mode of action of these treatments $[3,4]$. The discovery of molecular key defects that cause and/or drive the diseases however bears the promise for the development of potentially disruptive advanced therapeutics that are directed against the genetic and/or molecular origin of the disorder. The transformation of this knowledge for the patient's welfare however requires the integration of academic concepts and biotechnological-industrial capabilities beyond the current stage - a conceptual shortcoming that yet hinders the successful bench-to-bedside translation of numerous innovative discoveries. Among those therapeutic targets, however, that successfully transitioned from an academic concept to a biotechnological project, S100A1 emerged as a particular promising one given its unique molecular mode of action in cardiomyocytes, endothelial cells and on cardiac fibroblasts. Within cardiovascular cells, of which integrity and functions rely on the precise transduction of cytosolic and nuclear calcium $\left(\mathrm{Ca}^{2+}\right)$ signals, S100A1 operates as a superordinate regulator of $\mathrm{Ca}^{2+}$-controlled networks. In cardiomyocytes, S100A1 orchestrates and synchronizes, for example, the function and interplay of the sarcoplasmic reticulum (SR), sarcomere and mitochondria, respectively (previously reviewed in [5-8]). In heart failure, loss of its expression subsequently drives deterioration of systolic performance, disturbs diastolic relaxation, and promotes

'Molecular \& Translational Cardiology, Department of Internal Medicine III, Heidelberg University Hospital, Heidelberg University, INF 410, 69120 Heidelberg, Germany

${ }^{2}$ German Centre for Cardiovascular Research (DZHK), partner site Heidelberg/Mannheim, Heidelberg University Hospital, Heidelberg University, INF 410, 69120 Heidelberg, Germany

${ }^{3}$ Center for Translational Medicine, Department of Medicine, Thomas Jefferson University, Philadelphia, PA 19107, USA

${ }^{4}$ uniQure GmbH, INF 410, 69120 Heidelberg, Germany

*Author for correspondence: patrick.most@med.uni-heidelberg.de

\section{KEYWORDS}

- calcium cycling • cardiac fibroblast • endothelial cell dysfunction • gene therapy - heart failure $\bullet$ peripheral artery disease $\bullet$ S100A1 
cardiac remodeling and compromises energy homeostasis. In endothelial cells, S100A1 was shown to be a critical prerequisite, for example, for endoplasmic reticulum (ER) $\mathrm{Ca}^{2+}$ handling, $\mathrm{Ca}^{2+}$-activated nitric oxide (NO) generation and adaption of PKC signaling [9,10]. Lack of S100A1 entails endothelial dysfunction, results in disruption of vascular arterial and pulmonary relaxation and suppresses ischemia-driven angiogenesis. Besides its intracellular roles, S100A1 can be rapidly released from ischemic cardiomyocytes into the interstitial space and circulation. Internalized by adjacent cardiac fibroblasts, S100A1 activates an ER-located TLR signaling complex that contains innate immune system activation and tones down profibrotic reactions. Extracellular S100A1 thereby acts as an early molecular signal in the injured heart being most critical for balanced postischemic cardiac wound healing [11]. Figure 1 illustrates the role of S100A1 in different cardiac cell types and depicts corresponding pathophysiological conditions.

\section{S100A1 expression regulation \& pathophysiologies}

In the human heart, S100A1 is predominantly found in the left ventricle and mainly resides within cardiomyocytes among the different resident cardiac cell types. Subsequently lower concentrations in the right heart followed by left and right atria [13-17] display a pattern that most likely reflects the differential workload of each cardiac chamber. Progressively diminished S100A1 protein levels were characterized as a hallmark of chronically failing human myocardium $[16,18]$. Since S100A1 displays low abundance in the fetal heart but expression levels continuously rise until the heart is fully matured, it is likely that the loss of S100A1 protein in the failing heart is an integral part of the fetal gene program that is a pathological molecular signature of myocardial maladaptive remodeling and disease progression. Subsequent analyses in a variety of animal heart failure models, including rodents, rabbit and pig, corroborated the correlation of attenuated S100A1 expression with disease severity, progression and mortality [19-21].

Attempts to identify involved signaling pathways revealed transcriptional and subsequent translational suppression of the $S 100 A 1$ gene in cardiomyocytes in response to chronic in vitro G-protein coupled receptor stimulation, for example, via ET-1, Ang-II and phenylephrine. Direct PKC activation, which is downstream of the aforementioned pathways, likewise decreased S100A1 mRNA and protein levels, suggesting that $S 100 A 1$ downregulation in the course of maladaptive G-protein coupled receptor stimulation could be orchestrated, in part, through nuclear PKC actions [20]. Since S100A1 has been shown to inhibit PKC signaling, interruption of this negative feedback loop could promote an inhibitory vicious cycle that further accelerates the deleterious expression loss of the EF-hand $\mathrm{Ca}^{2+}$ sensor. Thus, clinically used drugs that shield the heart from chronically elevated Ang-II blood levels, might, at least in part, protect the heart against the detrimental diminution of S100A1 protein levels.

Refined expression analyses furthermore unveiled evidence for S100A1 abundance in mammalian and human macro- and microvascular endothelial cells of various organs [9,22-24]. Diminished S100A1 protein content was subsequently demonstrated in arterial endothelial cells of muscle biopsies from patients with critical limb ischemia due to severe peripheral artery disease [10]. Subsequent genetic ablation of S100A1 in endothelial cells entailed arterial hypertension and critical impairment of ischemic angiogenesis and neovascularization in mice $[9,10,22]$. Most recently, pulmonary hypertension was reported in mice lacking the $S 100 \mathrm{Al}$ gene, which corroborates the importance of the EF-hand $\mathrm{Ca}^{2+}$ sensor for the function of the intimal vascular cell layer [25].

Motivated by these findings, in vitro studies showed rapid loss of $S 100 A 1 \mathrm{mRNA}$ and protein levels in human endothelial cells both in response to hypoxia or inflammatory cytokines such as ET-1, Ang-II or TNFalpha [26,27]. A miRNAdependent mechanism was described that predominantly seems to regulate S100A1 abundance in endothelial cells. miR-138, which is upregulated by hypoxia and aforementioned cytokines, was found to target the $3^{\prime} \mathrm{UTR}$ of S100A1 mRNA, thereby leading to a rapid loss of $S 100 \mathrm{~A} 1$ protein in endothelial cells. However, inhibition of S100A1 protein levels by miR-138 could not be observed in various muscle cell types in vitro [26,27], indicating a cell-type-specific suppressive mechanism. Further studies are thus warranted to systematically decipher signaling cascades that negatively and positively control S100A1 transcription and translation in cardiac and vascular cell types.

Although cardiac fibroblasts neither express nor possess S100A1 protein, the EF-hand $\mathrm{Ca}^{2+}$ sensor is rapidly released from ischemic myocardium and detectable in cardiac fibroblasts adjacent to 


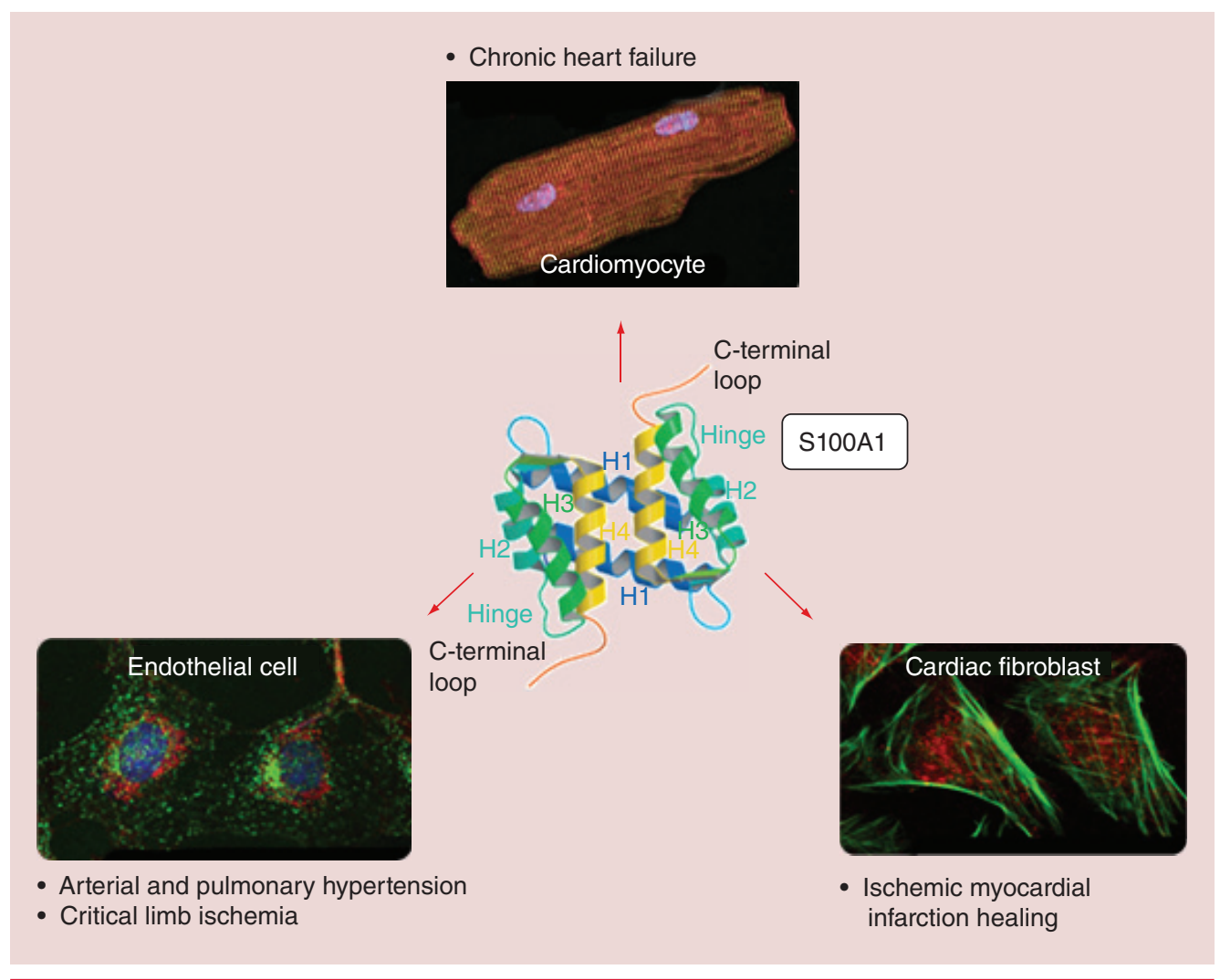

Figure 1. S100A1 in different cell types and corresponding cardiovascular pathophysiological conditions. Immunofluorescence images: adult rat cardiomyocyte: red-S100A1, green-ryanodine receptor 2, blue-DAPI/nucleus; mouse aorta endothelial cells: red-S100A1, green-endothelial NO synthase, blue-Draq5/nucleus; adult rat cardiac fibroblasts: red-internalized rhodamin-labeled S100A1, green-phalloidine/actin. Center: 3D picture of an S100A1 homodimer.

Reproduced with permission from [12].

damaged cardiomyocytes in ischemic myocardial tissue $[11,28]$. A most recent study indicated that extracellular S100A1 is specifically internalized by cardiac fibroblasts transforming them into an immune-modulatory and antifibrotic phenotype via an endosomal TLR4 signaling pathway that beneficially shapes the myocardial healing process [11].

\section{S100A1 molecular structure-function relationship}

S100A1, the prototypical cardiac S100 protein, belongs to the $S 100$ protein family, which is the largest subgroup of $\mathrm{Ca}^{2+}$ sensor proteins within the EF-hand $\mathrm{Ca}^{2+}$-binding superfamily. The S100A1 monomer has a molecular weight of approximately $10 \mathrm{kDa}$ [29] (Figure 2). Each monomer consists of two $\mathrm{Ca}^{2+}$-binding EF-hand motifs that are connected by a linker region (also referred to as hinge region). In each EF hand, the $\mathrm{Ca}^{2+}$-binding loop is flanked by $\alpha$-helices, so that helices I and II enclose the $\mathrm{N}$-terminal loop and helices III and IV the C-terminal loop, respectively [12,30-31]. Homodimerization of S100A1, which appears as the most prevalent quarternary structure within cells, occurs independent from $\mathrm{Ca}^{2+}$ binding in a symmetric and antiparallel manner that is stabilized through hydrophobic bonds between helices I and I' of each monomer. Upon $\mathrm{Ca}^{2+}$ binding to both EF hands, S100A1 undergoes a conformational change resulting in the exposure of a hydrophobic pocket [32]. The pocket forming domains, hinge region and the C-terminal extension, display the greatest sequence variability among the more than 20 different S100 isoforms.

The isoform-specific molecular moieties seem to determine the ability of S100A1 to engage with a specific subset of target proteins in a $\mathrm{Ca}^{2+}$-dependent manner in cardiac and vascular cells and to modulate their function allosterically [31]. $\mathrm{Ca}^{2+}$ binding of S100A1 appears 


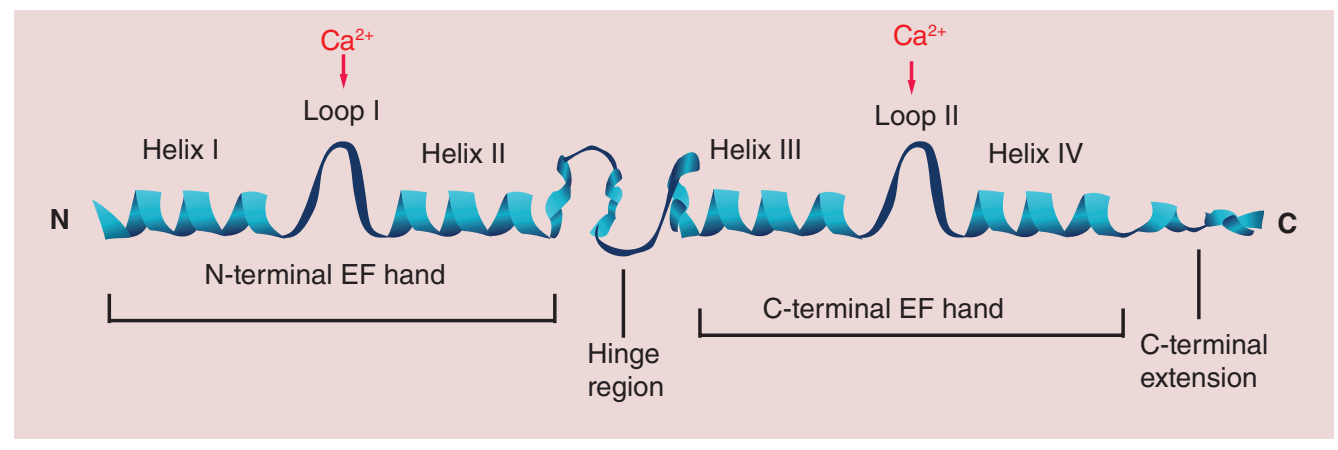

Figure 2. Schematic depiction of the secondary structure of an S100 protein. Each S100 monomer consists of a repetitive $\mathrm{Ca}^{2+}$-binding EF-hand motif. The $\mathrm{N}$-terminal (noncanonical) and C-terminal (canonical) EF hand are connected by a linker region (hinge region). The hinge region and C-terminal extension display the greatest sequence variability among individual members of the S100 protein family.

Reproduced with permission from [5], originally modified from [29].

to be regulated by post-translational modifications [33-35]. NO-dependent $S$-glutathionylation of the single cysteine residue located near the C-terminal extension (Cys85) seems to facilitate $\mathrm{Ca}^{2+}$ binding of both EF hands even at nanomolar free $\mathrm{Ca}^{2+}$ concentrations [33,35-36]. Moreover, S-nitrosylation at Cys86 favors a conformational change of both hinge region and C-terminal extension, potentially influencing target structure recognition [37]. Redox- and NO-dependent post-translational modifications might therefore empower intracellular S100A1 to decode spatially defined short-term as well as long-term global $\mathrm{Ca}^{2+}$ oscillations. Its $\mathrm{Ca}^{2+}$ sensing abilities might therefore account to link intrinsic calcium signaling with the $\mathrm{NO} /$ redox equilibrium [38].

\section{S100A1 in cardiomyocytes}

In ventricular cardiomyocytes, S100A1 resides in different subcellular compartments. A regular striated-like pattern dominates in myocardial tissue sections and cardiomyocytes (Figure 1, upper image) [19,39]. In part, this distribution pattern is due to interaction of $\mathrm{S} 100 \mathrm{~A} 1$ with target proteins located at the sarcoplasmic reticulum and the myofilaments [40-43]. Additionally, S100A1 is present in mitochondria where it is located both in the intermembraneous space and the matrix $[44,45]$.

\section{- Sarcoplasmic reticulum}

Several studies corroborated the colocalization as well as the physical interaction of S100A1 with the SERCA2a/PLB complex in human, rodent and pig cardiomyocytes $[19,46]$. Subsequent functional analyses of SR $\mathrm{Ca}^{2+}$ handling in intact cardiomyocytes, S100A1/SERCA2a co-expression studies in non-cardiac cell types and isolated SR vesicle preparations showed that binding of S100A1 to SERCA2a results in increased enzymatic activity, heightened sarcoplasmic reticulum (SR) $\mathrm{Ca}^{2+}$ uptake and enhanced SR $\mathrm{Ca}^{2+}$ load [20,47-48]. As S100A1 interaction with SERCA2a and PLB seems to be $\mathrm{Ca}^{2+}$-dependent, modulation of the SERCA2a/PLB complex by S100A1 most probably results from allosteric regulation. Within S100A1, the C-terminal amino acid residues of the protein (amino acids 75-94), which are part of the hydrophobic pocket exposed after $\mathrm{Ca}^{2+}$ binding, appear to convey the S100A1-SERCA2a interaction [19]. By sensing diastolic $\mathrm{Ca}^{2+}$ concentrations and subsequent SERCA2a stimulation, S100A1 might function as an endogenous opponent to PLB.

Importantly, in human failing cardiomyocytes with diminished SERCA2a abundance, reconstitution of diminished S100A1 expression by a genetically targeted intervention using an adenoviral vector that delivered the human S100A1 cDNA (Ad-S100A1) was sufficient to significantly elevate SERCA2a activity and accelerate cytoplasmic $\mathrm{Ca}^{2+}$ removal during diastole [21]. Hence, normalization of diastolic performance by S100A1 does not rely on normal SERCA2a protein levels. As a result, SR $\mathrm{Ca}^{2+}$ load was markedly heightened in Ad-S100A1-treated human failing cardiomyocytes when compared with Ad-control transfected cells. In response to $\beta$-adrenergic stimulation, both removal of cytosolic $\mathrm{Ca}^{2+}$ and $\mathrm{SR} \mathrm{Ca}{ }^{2+}$ load were increased, reflecting not only preserved but improved 
$\beta$-adrenergic responsiveness. Therapeutic rescue of diastolic function did not require the normalization of aberrant PLB phosphorylation at Ser16 or Thr17. This suggests that S100A1 most likely operates independent and downstream of cAMP-dependent protein kinase $\mathrm{A}$ and $\mathrm{Ca}^{2+} /$ calmodulin-dependent protein kinase II activity [21]. S100A1 thereby targets cardiomyocyte $\mathrm{Ca}^{2+}$ cycling and contractile performance through direct modulation of SERCA2a activity bypassing the defective components of the $\beta$-adrenergic signaling pathway.

Further studies have demonstrated that S100A1 can modulate RyR2 function under both diastolic and systolic conditions [6,19,21,46,49]. During diastole, S100A1 decreased both frequency and amplitude of $\mathrm{SR} \mathrm{Ca}{ }^{2+}$ events $\left(\mathrm{Ca}^{2+}\right.$ sparks) in quiescent permeabilized rat cardiomyocytes [49]. Under systolic conditions, S100A1 increased SR $\mathrm{Ca}^{2+}$ release and excitation-contraction coupling gain in voltage-clamped rabbit cardiomyocytes [46]. Accordingly, gene-therapy-mediated S100A1 reconstitution in human failing cardiomyocytes resulted in significantly improved fractional shortening [21]. Importantly, the negative force-frequency response, which is a hallmark of human heart failure, was reversed by Ad-S100A1 treatment corroborating normalized $\mathrm{Ca}^{2+}$ handling both under systolic and diastolic conditions. In line with reduced RyR 2 open probability at diastolic $\mathrm{Ca}^{2+}$ concentrations, Ad-S100A1 treatment increased SR Ca ${ }^{2+}$ load but prevented human failing cardiomyocytes from arrhythmogenic diastolic SR $\mathrm{Ca}^{2+}$ leak in response to $\beta$-adrenergic stimulation.

Therapeutic impact of $S 100 A 1$ re-expression on disturbed SERCA2a and RyR2 function in failing cardiomyocytes was eventually confirmed in large animal studies approximating human cardiovascular anatomy and pathophysiology [50,51]. Overall, current data point towards a cAMP-independent and synergistic allosteric regulation both of SERCA2a and RyR2 through $\mathrm{Ca}^{2+}$-activated S100A1 during the contractile cycle. Further delineation of S100A1's molecular structure-function relationship with its SR target proteins is subject of ongoing research.

\section{- Mitochondria}

Ultrastructural analyses by the use of confocal and immuno-electron microscopy lead to the detection of S100A1 within mitochondria of cardiomyocytes $[44,45]$. Further studies identified mitochondrial F1/0-ATPase, isocitrate dehydrogenase and adenine nucleotide translocator (ANT) amongst others as mitochondrial targets of S100A1 [44]. $\mathrm{Ca}^{2+}$-dependent interaction of S100A1 with mitochondrial F1/0-ATPase resulted in stimulation of ATP synthase activity, suggesting a fundamental role of S100A1 in the regulation of cardiac energy homeostasis. S100A1 binding to ANT indicates a possible involvement in the ADP/ATP exchange process between the mitochondrial matrix and the cytoplasm in cardiomyocytes. Interaction of the EF-hand $\mathrm{Ca}^{2+}$ sensor with $\mathrm{Ca}^{2+}$-regulated key enzymes of the citrate cycle such as isocitrate dehydrogenase predicts an additional role in NADH synthesis. Accordingly, diminished mitochondrial ATPase activity and decreased ATP levels were found in S100A1-deficient rat and human failing cardiomyocytes $[19,21,44]$. Adenoviral reconstitution of S100A1 (Ad-S100A1) resulted in significantly elevated phosphocreatine/ATP and NADH/ NAD ratios [21], of which the former is an independent predictor of heart failure mortality [52]. Functional analyses of mitochondria isolated from Ad-S100A1-treated human failing cardiomyocytes revealed restored mitochondrial S100A1 content associated with superior electron flow. Moreover, Ad-S100A1 prevented mitochondrial transmembrane potential depolarization and permeability transition under both basal conditions and in response to $\mathrm{Ca}^{2+}$ overload. This indicates that compromized energy homeostasis and mitochondrial function in failing cardiomyocytes may be ameliorated through S100A1 gene replacement. Nevertheless, further studies are needed for a holistic understanding of S100A1's beneficial role in cardiac energy homeostasis and whether this effects also extends, for example, to endothelial cells.

\section{- Sarcomere}

S100A1 has furthermore been detected at several sites within the sarcomere [14-15,40-43]. The use of immuno-electron microscopy uncovered S100A1 binding to the Z-line, periphery of M-lines as well as I- and A-bands. Subsequent analyses unveiled the giant myofilament protein titin as S100A1 binding partner and identified three distinctive interaction sites within its passive force generating region [43]. $\mathrm{Ca}^{2+}$-dependent S100A1 interaction with the PEVK subdomain was then reported to result in improved sarcomeric compliance potentially by reducing the force that arises as F-actin slides relative to the PEVK domain. S100A1-titin interaction 
might therefore result in diminished pre-contractile passive tension in cardiomyocytes [43,53]. Additionally, S100A1 reduces myofilament $\mathrm{Ca}^{2+}$ sensitivity and co-operativity without affecting troponin I phosphorylation or maximal force development [47]. Thus, $\mathrm{Ca}^{2+}$-dependent S100A1 interplay with components of the sarcomere could facilitate cardiac relaxation improving myofilament mechanics and diastolic dissociation of $\mathrm{Ca}^{2+}$, which potentially synergizes with its impact on diastolic SERCA2a and RyR2 activity. Further investigation of its potential impact on other sarcomeric structures is motivated by its profound but yet mechanistically unresolved antihypertrophic actions in remodeled myocardium in vivo [42].

In summary, cytoplasmic S100A1 seems to orchestrate cardiomyocyte $\mathrm{Ca}^{2+}$ homeostasis as a superordinate regulator of downstream factors that operate as gatekeepers of the $\mathrm{Ca}^{2+}$ handling and contractile machinery and cardiac energy metabolism. Lack of S100A1 in cardiomyocytes appears to promote a system's failure that drives progression and mortality of the disease. Targeted restoration of S100A1 in cardiomyocytes restores function and interplay of several subsystems, thereby mediating long-term restoration of systolic and diastolic cardiac performance in human relevant animal models. Figure 3 displays the current conceptual framework of S100A1 targets and mechanisms in cardiomyocytes.

\section{S100A1 in vascular endothelial cells}

In both macro- and microvascular endothelial cells, S100A1 is mainly dispersed in a granular pattern throughout the cytoplasm with perinuclear enrichment [9]. The use of immunohistochemistry and confocal microscopy disclosed the presence of S100A1 at the ER. Alike its interaction with SERCA2a and RyR2 in cardiomyocytes, S100A1 targets SERCA and $\mathrm{IP}_{3} \mathrm{R}$ as key effectors of $\mathrm{ER} \mathrm{Ca}^{2+}$ uptake and release in endothelial cells $[7,10]$. Consequently, endothelial cells isolated from $S 100 A 1$ knock out mice (SKO) exhibited reduced $\mathrm{Ca}^{2+}$ transients in response to muscarinergic receptor stimulation. In line with these findings, siRNA-mediated S100A1 knockdown resulted in significantly decreased kininergic receptor-induced cytoplasmic $\mathrm{Ca}^{2+}$ peak formation. In further support of a critical role of S100A1 in $\mathrm{IP}_{3}$-mediated $\mathrm{Ca}^{2+}$ signaling, adenoviral S100A1 expression in SKO endothelial cells normalized $\mathrm{Ca}^{2+}$ transients in response to both acetylcholine and bradykinin. Extending the range of S100A1 targets, co-immunoprecipitation of eNOS indicated a direct $\mathrm{Ca}^{2+}$-dependent binding of S100A1 to eNOS [10]. Subsequent analyses linked disturbed $\mathrm{Ca}^{2+}$ signaling in $\mathrm{SKO}$ endothelial cells to impaired NO production at baseline and attenuated responsiveness to acetylcholine, bradykinin and thrombin $[9,22,54]$. Diminished NO generation occurred at comparable expression levels of eNOS in S100A1 knockdown endothelial cells, pointing towards a conclusive regulatory mechanism. In line with these results, S100A1 overexpression in human coronary artery endothelial cells resulted in increased NO release upon stimulation with acetylcholine.

Indicating pathophysiological relevance, the dysfunctional S100A1-deficent intimal layer caused both arterial and pulmonary hypertension in mice due to impaired relaxation of arterial vessels in response to various endogenous vasodilators $[10,25]$. Moreover, analysis of gastrocnemius muscle tissue from patients with critical limb ischemia showed that endothelial cell S100A1 mRNA levels were markedly reduced [10]. Corroborating evidence was obtained in an experimental hind limb ischemia model in $\mathrm{SKO}$ mice. This resulted in a high rate of limb loss due to defective postischemic angiogenesis, whereas perfusion of ischemic limbs recovered in wild-type animals [10]. S100A1-deficient human microvascular ECs displayed attenuated NO generation in response to VEGF and hypoxia, and showed diminished proliferation, lower migration rates and impaired capillary-like tube formation in vitro. In subsequent biochemical analyses, VEGF stimulation resulted in hyperphosphorylation of the inhibitory eNOS Thr495 site in S100A1-deficient ECs, whereas controls demonstrated enhanced phosphorylation of the stimulatory Ser1177 residue. In this study, inhibitory eNOS Thr495-phosphorylation in S100A1-deficient ECs was dependent on heightened PKC activity. Importantly, genetically mediated elevation of S100A1 protein levels attenuated PKC activity. In addition to altered VEGF-signaling, co-immunoprecipitation, immunofluorescence co-localization and proximity ligation assays revealed a $\mathrm{Ca}^{2+}$-dependent direct regulatory S100A1-eNOS interaction. In vivo, an inhibitory eNOS phosphorylation pattern was observed together with defective VEGF signaling in ischemic limbs of SKO mice. Further corroborating the central role of 


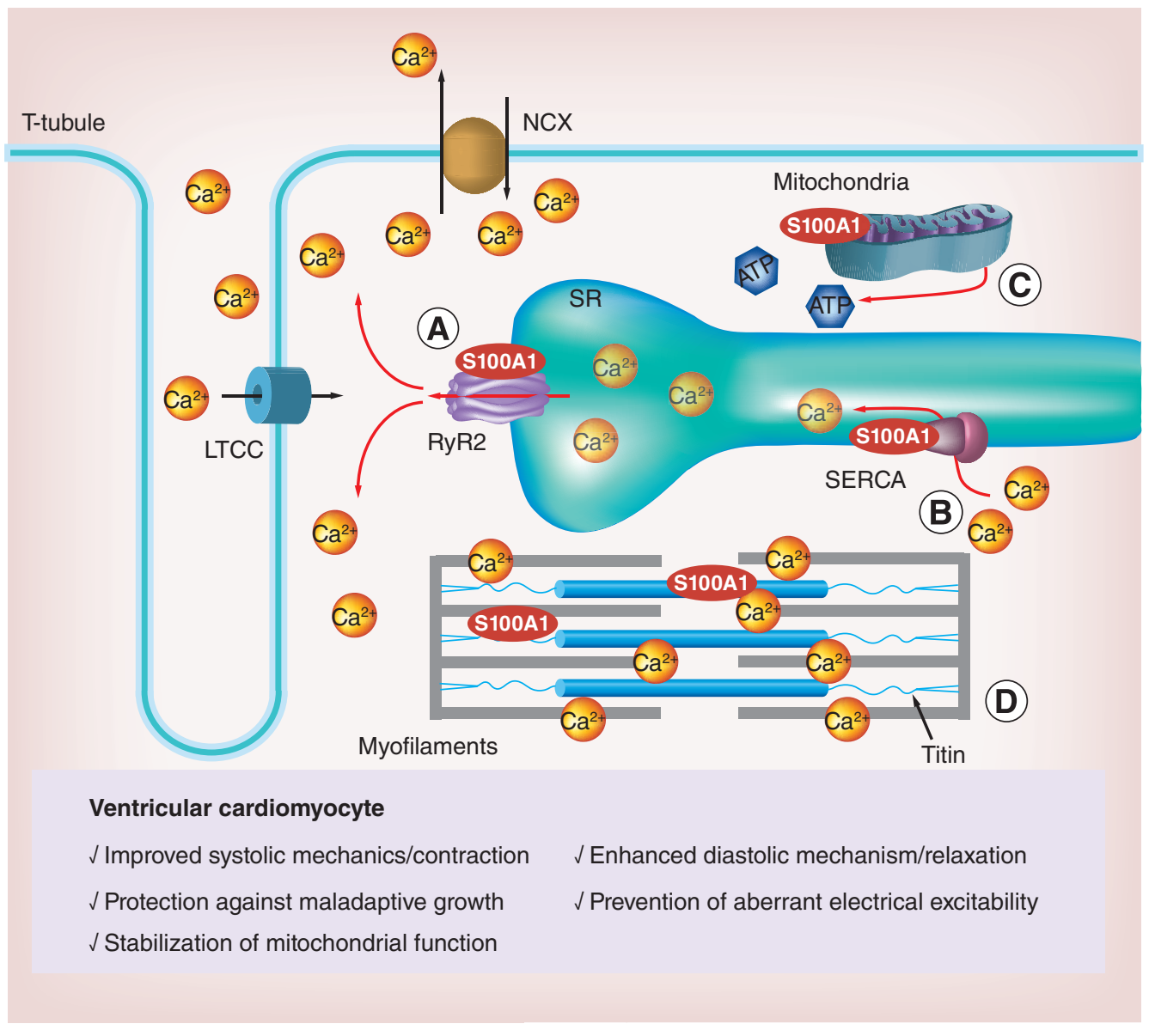

Figure 3. Conceptual framework of S100A1 target structures in cardiomyocytes. (A) S100A1 protein interacts with RyR2, resulting in enhanced systolic SR Ca ${ }^{2+}$ release, improved EC-coupling gain and prevention of arrhythmogenic diastolic $\mathrm{Ca}^{2+}$ leakage. (B) Augmented systolic $\mathrm{SR} \mathrm{Ca}{ }^{2+}$ release is compensated by elevated SERCA2a activity, leading to accelerated removal of cytosolic $\mathrm{Ca}^{2+}$ and increased SR Ca ${ }^{2+}$ load. (C) S100A1 interaction with mitochondria enhances mitochondrial ATP retrieval, matching increased energy demands. (D) S100A1 interference with titin-actin interplay decreases myofilament stiffness and facilitates diastolic $\mathrm{Ca}^{2+}$ dissociation, alleviating passive tension and diastolic relaxation of the contractile apparatus.

LTCC: L-type calcium channel; NCX: Sodium-calcium exchanger; RyR2: ryanodine receptor 2;

SERCA: Sarcoplasmic/endoplasmic reticulum calcium ATPase; SR: Sarcoplasmic reticulum.

Reproduced with permission from [6].

deficient NO bioavailability in the post-ischemic SKO phenotype, treatment with the NO donor DETA-NO prevented loss of ischemic hind limbs in SKO mice.

In summary, these results provide evidence for a critical role of S100A1 in EC NO homeostasis with relevance for arterial and pulmonary vascular tone control and postnatal ischemia-driven angiogenesis. Originating from S100A1 expression changes in patients with defective arterial limb perfusion, S100A1 targeting of $\mathrm{Ca}^{2+}$ - and PKC-dependent networks together with direct stimulatory eNOS interaction has been shown to represent an indispensable prerequisite to mount an adaptive angiogenic response to ischemia. Nonetheless, possible interactions of S100A1 with other $\mathrm{Ca}^{2+}$-dependent signaling pathways in ECs remain to be elucidated. Furthermore, EC-specific downregulation of S100A1 by miR138 provides the rationale for studies investigating miR-138 as novel therapeutic target for EC dysfunction (see section S100A1 expression regulation). Restoration of $S 100 A 1$ expression in endothelial cells rescued miR-138-mediated endothelial cell dysfunction pointing towards a therapeutic potential of targeting miR-138 in 


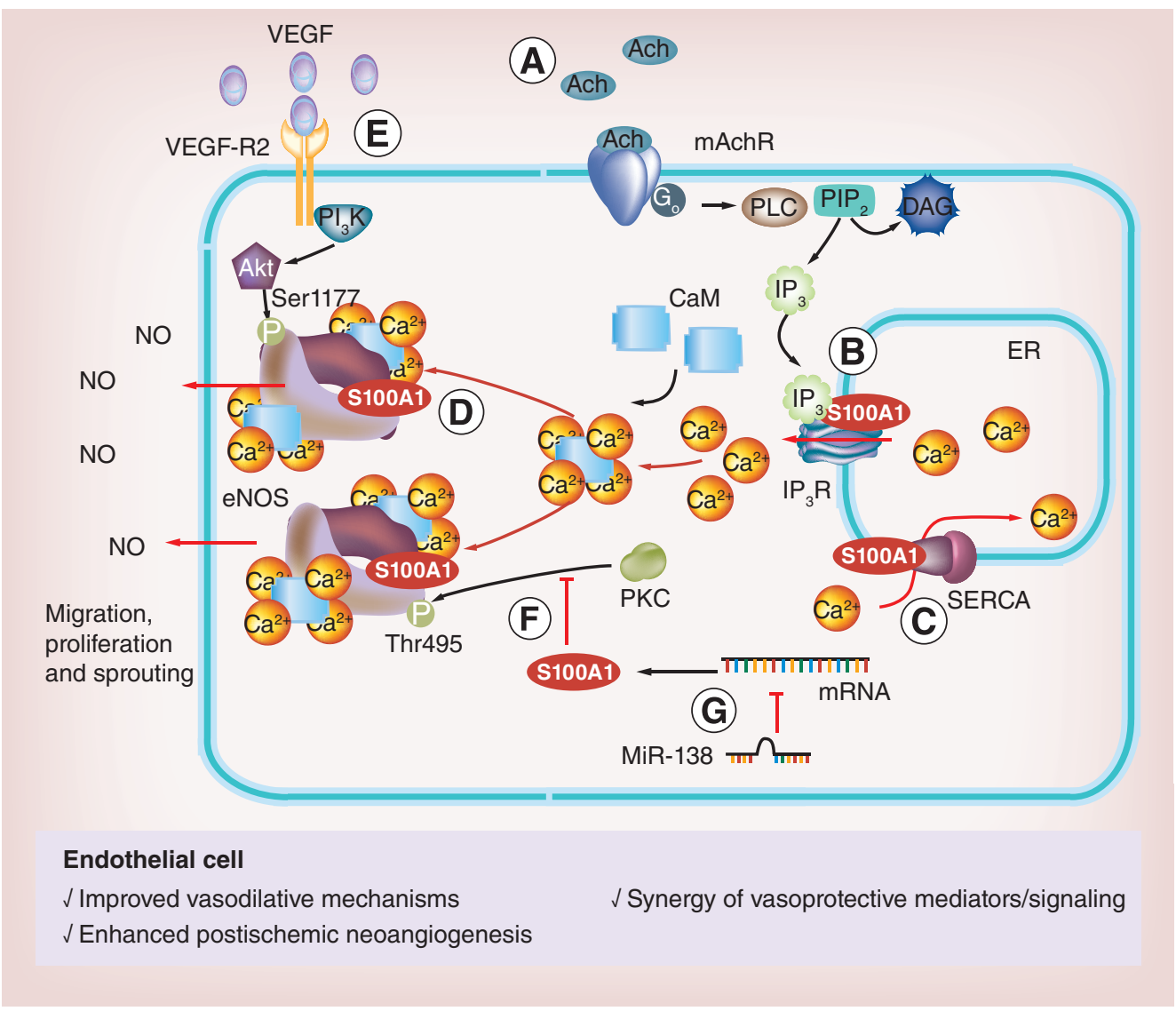

Figure 4. Proposed model for S100A1 molecular interactions in endothelial cells. (A) mAchR stimulation results in inositol-1,4,5-triphosphate $\left(\mathrm{IP}_{3}\right)$-mediated $E R \mathrm{Ca}^{2+}$ release. S100A1 interaction with $I P_{3} R(B)$ and SERCA (C) enhance ER $\mathrm{Ca}^{2+}$ release which is required for eNOS activation by $\mathrm{Ca}^{2+}$ / CaM. (D) Direct binding of S100A1 to eNOS further potentiates eNOS enzymatic activity, leading to augmented NO production and EC migration, proliferation and sprouting. (E) Extracellular VEGF stimulates the VEGF-R2/PI $\mathrm{K} / \mathrm{Akt}$ axis, resulting in eNOS activation due to enhanced Akt-mediated Ser1177 phosphorylation. (F) S100A1 prevents PKC-mediated inhibitory eNOS hyperphosphorylation at Thr495 in response to VEGF. (G) In endothelial cells, S100A1 translation is suppressed by MiR-138. Ach: Acetylcholine; CaM: calmodulin; DAG: Diacyl-glycerol; eNOS: Endothelial nitric oxide synthase; ER: Endoplasmic reticulum; IP R: IP3-receptor; mAchR: Muscarinergic Gq-protein coupled receptor; MiR-138: microRNA-138; PKC: Protein kinase C; PLC: Phospholipase C; SERCA: Sarcoplasmic/ endoplasmic reticulum calcium ATPase.

Reproduced with permission from [5].

vascular endothelium [26,27]. Figure 4 proposes a model for S100A1 molecular interactions in endothelial cells.

\section{Extracellular S100A1 targeting cardiac} fibroblasts

Elevated S100A1 serum levels were reported in patients after open heart surgery and myocardial infarction [55,56] and rapid depletion of S100A1 was subsequently demonstrated in ischemic rat and human myocardium [28]. Among the proteins passively released from damaged cells, distinct molecules may play an active role in the restoration of tissue homeostasis [57]. These molecules are referred to as damage-associated molecular patterns or 'alarmins' and include the prototypical HMGB1 and members of the $\$ 100$ protein family (reviewed in [58-60]).

Originating from novel clinical data showing transient S100A1 release in patients with acute myocardial infarction, a most recent study examined the biological effect of extracellular S100A1 in the injured heart [11]. While cardiac fibroblasts (CFs) do not express endogenous 
S100A1, they stained positive for intracellular S100A1 when adjacent to damaged cardiomyocytes in a murine model of experimental myocardial infarction [11]. In support of a cardiomyocyte origin, cultured CFs subjected to S100A1-containing supernatant from necrotic cardiomyocytes stained positive for intracellular S100A1 in vitro. Comparable S100A1 internalization could neither be detected in adult cardiomyocytes nor in smooth muscle or endothelial cells, suggesting a CF-specific effect. In CFs, delivery of rhodamine labeled (rho)-S100A1 to acidic endolysosomes and subsequent transient activation of MAPK/ SAPK and NF-kappaB signaling was dependent on binding of S100A1 to endosomal TLR 4. Examined at the level of gene and protein expression, S100A1 internalization resulted in an immunomodulatory and antifibrotic phenotype transition of CFs. Comparable transcriptional changes were observed after intramyocardial injection of S100A1 in vivo. Systemic application of an S100A1-neutralizing antibody prior to experimental myocardial infarction led to significantly increased myocardial infarct size and impaired postischemic left ventricular function. Analysis of myocardial gene expression at time points ranging from $3 \mathrm{~h}$ to 7 days post-infarction revealed prolonged myocardial inflammation in the anti-S100A1 treated group.

Taken together, these studies ascribe beneficial cardiac alarmin properties to S100A1 released from necrotic cardiomyocytes, principally opposing the previously reported detrimental actions of HMGB1 [61]. By targeting cardiac fibroblasts, S100A1 may act as a direct

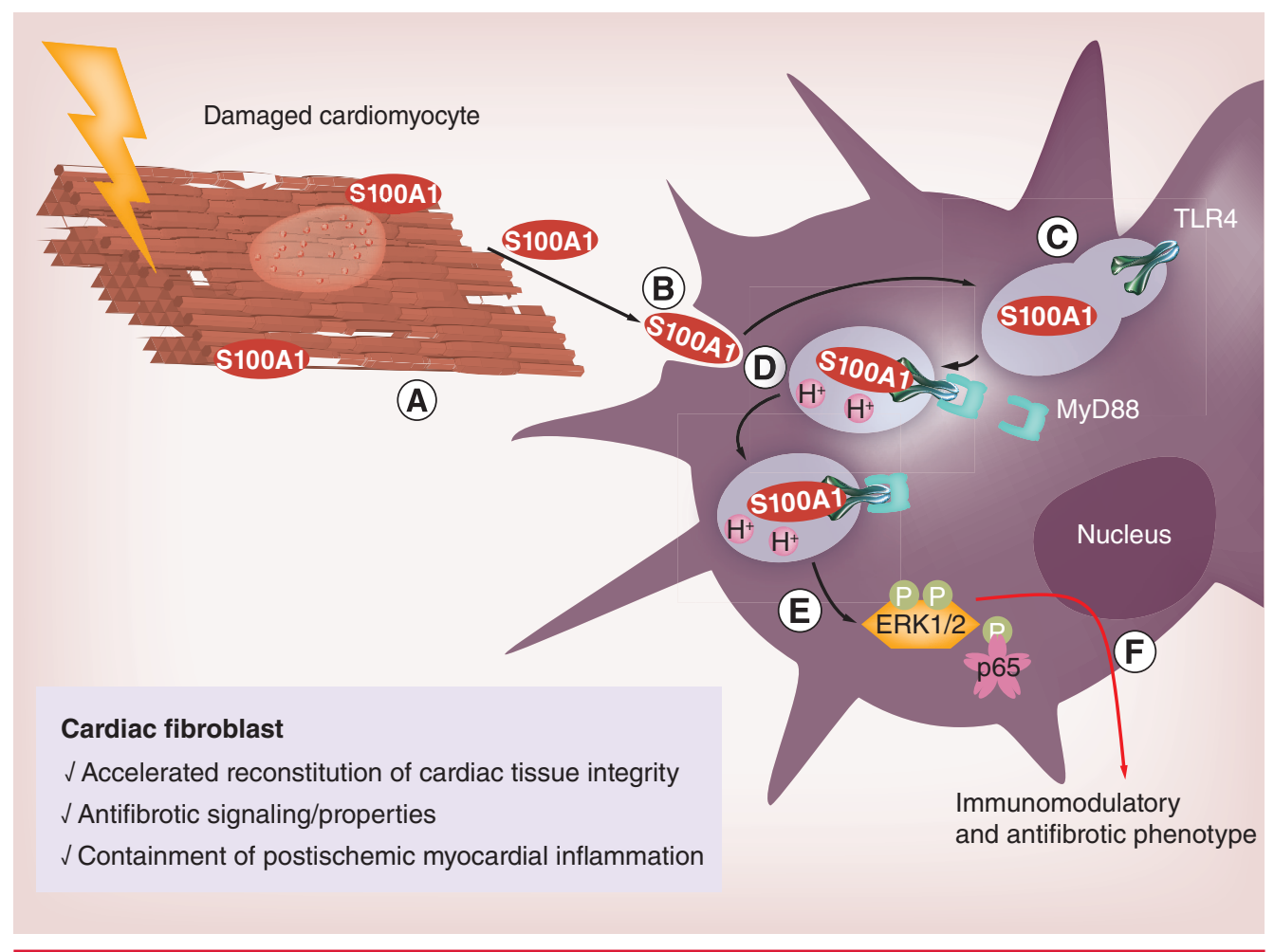

Figure 5. Schematic illustration of extracellular S100A1 from damaged cardiomyocytes targeting cardiac fibroblasts. (A) Ischemic injury leads to release of S100A1 from cardiomyocytes into the myocardial interstitium. (B) Extracellular S100A1 is specifically internalized by cardiac fibroblasts through clathrin- and caveolin-independent fluid endocytosis. (C) Endocytosed S100A1 binds to intracellular TLR4, thereby prompting trafficking to acidified endolysosomes. (D) MyD88 binds to the cytoplasmic domain of acidified TLR4 and initiates signal transduction. (E) The S100A1-TLR4-MyD88 signaling complex transiently activates MAPK/SAPK and NF- $\kappa B$ pathways. (F) S100A1-mediated signal transduction leads to an antifibrotic and immunomodulatory phenotype transition of cardiac fibroblasts.

Modified with permission from [11]. 
molecular link between cardiomyocyte death and beneficial post-ischemic inflammation and healing. However, the potentially beneficial role of extracellular S100A1 in myocardial damage repair warrants further investigation. Figure 5 gives a schematic overview on our current understanding of extracellular S100A1 targeting cardiac fibroblasts.

Conclusion \& translational perspective Taking advantage of S100A1's pleiotropic molecular profile in cardiomyocytes, adeno-associated viral (AAV-S100A1) S100A1 gene therapy of chronic heart failure is being developed toward a first-in-men clinical trial (recently reviewed in $[62,63])$. Key to the successful translation was the combination of in-depth understanding of the therapeutic mode of action in human-relevant large animal disease models with access to a scalable and modular biotechnological AAV production platform and respective regulatory expertise for gene therapeutic medicinal products (GTMPs). Profound long-term therapeutic actions of AAV-S100A1 together with predictive biomarkers and full compatibility with standard heart failure treatment bear the potential for a truly disruptive theranostic that can change the landscape of future chronic heart failure therapy. Due to similar S100A1 dysregulation in rodent models of right ventricular pressure overload (own unpublished data) and diastolic failure [64], translational studies in appropriate large animal disease models are underway to answer the question whether beneficial effects both on systolic and diastolic mechanisms by cardiac-targeted S100A1 gene replacement extend to Cor pulmonale and heart failure with preserved left ventricular ejection fraction. To

\section{EXECUTIVE SUMMARY}

\section{S100A1 expression regulation}

- Cardiomyocyte S100A1 expression is diminished in heart failure due to prohypertrophic stimuli via GPCRs/PKC and occurs most likely as an integral part of fetal gene reprogramming in the course of adverse remodeling. In endothelial cells, S100A1 downregulation occurs in response to inflammatory cytokines via miR-138. While cardiac fibroblasts do not express $\mathrm{S100A1}$, they are capable of internalizing significant amounts of the EF-hand $\mathrm{Ca}^{2+}$ sensor upon release from damaged cardiomyocytes.

\section{S100A1 molecular structure-function relationship}

- Each S100A1 monomer contains two $\mathrm{Ca}^{2+}$-binding domains most likely favoring a quarternary homodimeric structure inside cells. Upon $\mathrm{Ca}^{2+}$ binding, hydrophobic isoform-specific molecular moieties being exposed to the molecular surface are thought to convey target recognition and modulation of its activity.

\section{S100A1 in cardiomyocytes}

- S100A1 acts as a superordinate regulator of cardiomyocyte $\mathrm{Ca}^{2+}$ homeostasis orchestrating and synchronizing the function of downstream key regulators of SR (e.g. SERCA2a, RyR2), sarcomere (e.g. cardiac titin) and mitochondrial (e.g. F1/0-ATPase) function. Targeted replacement of its expression loss in chronic heart failure is a disruptive therapeutic concept for long-term restoration of systolic and diastolic performance in men.

\section{S100A1 in vascular endothelial cells}

- In endothelial cells, S100A1 is involved in NO homeostasis and $\mathrm{Ca}^{2+}$ handling regulation, targeting, for example, the eNOS/PKC interactome as well as IP3R and SERCA function. Suppression of intimal S100A1 expression such as in peripheral artery disease compromises ischemia-driven angiogenesis and has been linked to arterial and pulmonary hypertension.

\section{Extracellular S100A1 targeting cardiac fibroblasts}

- S100A1, which is released from damaged cardiomyocytes, modulates the cardiac fibroblast phenotype and beneficially impacts post-ischemic inflammation and healing operating as a novel cardiac alarmin.

\section{Conclusion \& translational perspective}

- AAV-S100A1 gene therapy of chronic heart failure is close to first-in-human clinical trials. Further studies will have to evaluate the therapeutic potential of S100A1-based molecular interventions in further cardiac and vascular disease and acute myocardial infarction. 
exploit S100A1's molecular actions in endothelial cells targeted delivery modes are yet to be developed to design S100A1-based treatments of diseases that are caused by endothelial dysfunction such as arterial and pulmonary hypertension, peripheral and coronary artery disease and atherosclerosis. The most recent discovery of cardiac alarmin properties of damage-released S100A1 protein in the heart [11] might stimulate strategies opting at improved healing of infarcted myocardium.

\section{Future perspective}

By 2020 it is expected that the AAV-S100A1 treatment of chronic heart failure will emerge as the best-in-class GTMP for chronic heart failure due to a superior mode of action and respective companion diagnostics. Further advancement in synthetic vector technology and gene expression regulation is expected to drive the second generation of S100A1-based heart failure GTMPs. Therapeutic concepts enabling targeted manipulation of S100A1 concentrations in Cor pulmonale and heart failure with preserved left ventricular ejection fraction might have closed the gap to first first-in-human trials. Availability of endothelial-targeted formulations might have fostered the generation of proof-of-concept studies on S100A1-based reversal of endothelial dysfunction in human-relevant disease models.
Large-scale production of human recombinant S100A1 protein is expected to provide the ground for studies testing its potential to beneficially shape the myocardial infarction healing process by limiting inflammation-associated myocardial damage.

\section{Financial \& competing interests disclosure}

$P$ Most and HA Katus hold patents on the therapeutic use of S100A1 in cardiovascular diseases. P Most and H Katus are shareholders of uniQure NK. P Most is managing director of uniQure GmbH Germany. This work was funded by a post-doctoral research grant from the Ernst und Berta Grimmke Foundation (to D Rohde), an Oskar Lapp Stipend awarded by the German Cardiac Society (DGK; to D Rohde), NIH grants R01 HL07842 (to K Peppel) and R01 HL092130-01 and HL092130-02S1 (to P Most), Perspektivförderung des Landes Baden-Württemberg (to $P$ Most and HA Katus) and grants from the German Centre for Cardiovascular Research (DZHK; to P Most and HA Katus), the German Federal Ministry of Education and Research (BMBF; to P Most and HA Katus) and the German Research Foundation (DFG; to P Most). The authors have no other relevant affiliations or financial involvement with any organization or entity with a financial interest in or financial conflict with the subject matter or materials discussed in the manuscript apart from those disclosed.

No writing assistance was utilized in the production of this manuscript.

\section{References}

Papers of special note have been highlighted as: - of interest; $\bullet \bullet$ of considerable interest.

1 Organization WHO. Fact sheet No 310. (2014).

www.who.int/mediacentre/factsheets/fs310

2 Go AS, Mozaffarian D, Roger VL et al. Heart disease and stroke statistics - 2014 update: a report from the American Heart Association. Circulation 129(3), e28-e292 (2014).

3 McMurray JJ, Adamopoulos S, Anker SD et al. ESC Guidelines for the diagnosis and treatment of acute and chronic heart failure 2012: The Task Force for the Diagnosis and Treatment of Acute and Chronic Heart Failure 2012 of the European Society of Cardiology. Developed in collaboration with the Heart Failure Association (HFA) of the ESC. Eur. Heart J. 33(14), 1787-1847 (2012).

4 Stewart S, MacIntyre K, Hole DJ, Capewell S, McMurray JJ. More 'malignant' than cancer? Five-year survival following a first admission for heart failure. Eur. J. Heart Fail. 3(3), 315-322 (2001).
5 Rohde D, Ritterhoff J, Voelkers M, Katus HA, Parker TG, Most P. S100A1: a multifaceted therapeutic target in cardiovascular disease. J. Cardiovasc. Transl. Res. 3(5), 525-537 (2010).

6 Volkers M, Rohde D, Goodman C, Most P. S100A1: a regulator of striated muscle sarcoplasmic reticulum $\mathrm{Ca}^{2+}$ handling, sarcomeric, and mitochondrial function. J. Biomed. Biotechnol. 2010, 178614 (2010).

7 Kraus C, Rohde D, Weidenhammer C et al. S100A1 in cardiovascular health and disease: closing the gap between basic science and clinical therapy. J. Mol. Cell Cardiol. 47(4), 445-455 (2009).

8 Rohde D, Brinks H, Ritterhoff J, Qui G, Ren S, Most P. S100A1 gene therapy for heart failure: a novel strategy on the verge of clinical trials. J. Mol. Cell. Cardiol. 50(5), 777-784 (2011)

9 Pleger ST, Harris DM, Shan C et al. Endothelial S100A1 modulates vascular function via nitric oxide. Circ. Res. 102(7), 786-794 (2008).
- $\quad$ First study presenting S100A1 as a modulator of endothelial cell function.

10 Most P, Lerchenmuller C, Rengo G et al. S100A1 deficiency impairs postischemic angiogenesis via compromised proangiogenic endothelial cell function and nitric oxide synthase regulation. Circ. Res. 112(1), 66-78 (2013).

-• First study linking endothelial cell dysfunction due to S100A1 downregulation to impaired ischemic angiogenesis and neovascularization.

11 Rohde D, Schon C, Boerries M et al. S100A1 is released from ischemic cardiomyocytes and signals myocardial damage via Toll-like receptor 4. EMBO Mol. Med. 6(6), 778-794 (2014).

- First study demonstrating alarmin properties for S100A1 from necrotic cardiomyocytes.

12 Rustandi RR, Baldisseri DM, Inman KG et al. Three-dimensional solution structure of the calcium-signaling protein apo-S100A1 as 
determined by NMR. Biochemistry 41(3), 788-796 (2002).

13 Ehlermann P, Remppis A, Guddat O et al. Right ventricular upregulation of the $\mathrm{Ca}^{2+}$ binding protein S100A1 in chronic pulmonary hypertension. Biochim. Biophys. Acta 1500(2), 249-255 (2000).

14 Haimoto H, Kato K. S100a0 (alpha alpha) protein in cardiac muscle. Isolation from human cardiac muscle and ultrastructural localization. Eur. J. Biochem. 171(1-2), 409-415 (1988).

15 Kato K, Kimura S, Haimoto H, Suzuki F. S100a0 (alpha alpha) protein: distribution in muscle tissues of various animals and purification from human pectoral muscle. J. Neurochem. 46(5), 1555-1560 (1986).

16 Remppis A, Greten T, Schafer BW et al. Altered expression of the $\mathrm{Ca}(2+)$-binding protein S100A1 in human cardiomyopathy. Biochim. Biophys. Acta 1313(3), 253-257 (1996).

17 Zimmer DB, Song W, Zimmer WE. Isolation of a rat $S 100$ alpha cDNA and distribution of its mRNA in rat tissues. Brain Res. Bull. 27(2), 157-162 (1991).

18 Bennett MK, Sweet WE, Baicker-McKnee S et al. S100A1 in human heart failure: lack of recovery following left ventricular assist device support. Circ. Heart Fail. 7(4), 612-618 (2014).

19 Most P, Pleger ST, Volkers M et al. Cardiac adenoviral S100A1 gene delivery rescues failing myocardium. J. Clin. Invest. 114(11), 1550-1563 (2004).

20 Most P, Seifert H, Gao E et al. Cardiac S100A1 protein levels determine contractile performance and propensity toward heart failure after myocardial infarction. Circulation 114(12), 1258-1268 (2006).

21 Brinks H, Rohde D, Voelkers M et al. S100A1 genetically targeted therapy reverses dysfunction of human failing cardiomyocytes. J. Am. Coll. Cardiol. 58(9), 966-973 (2011).

-• First study demonstrating therapeutic efficacy of S100A1 expression in human failing cardiomyocytes.

22 Desjardins JF, Pourdjabbar A, Quan A et al. Lack of S100A1 in mice confers a genderdependent hypertensive phenotype and increased mortality after myocardial infarction. Am. J. Physiol. Heart Circ. Physiol. 296(5), H1457-H1465 (2009).

23 Desjardins JF, Teichert-Kuliszewska K, Parker T. S100A1: a pluripotent regulator of cardiac and vascular function. Can. J. Cardiol. 26(Suppl. A) 9A-12A (2010).
24 Lefranc F, Decaestecker C, Brotchi J et al. Co-expression/co-location of $\mathrm{S} 100$ proteins (S100B, S100A1 and S100A2) and protein kinase $\mathrm{C}$ (PKC-beta, -eta and -zeta) in a rat model of cerebral basilar artery vasospasm. Neuropathol. Appl. Neurobiol. 31(6), 649-660 (2005).

25 Teichert-Kuliszewska K, Tsoporis JN, Desjardins JF et al. Absence of the calciumbinding protein, S100A1, confers pulmonary hypertension in mice associated with endothelial dysfunction and apoptosis. Cardiovasc. Res. 105(1), 8-19 (2015).

26 Sen A, Ren S, Lerchenmuller C et al. MicroRNA-138 regulates hypoxia-induced endothelial cell dysfunction by targeting S100A1. PLoS ONE 8(11), e78684 (2013).

27 Sen A, Most P, Peppel K. Induction of microRNA-138 by pro-inflammatory cytokines causes endothelial cell dysfunction. FEBS Lett. 588(6), 906-914 (2014).

28 Bi H, Yang Y, Huang J, Li Y, Ma C, Cong B. Immunohistochemical detection of S100A1 in the postmortem diagnosis of acute myocardial infarction. Diagn. Pathol. 8, 84 (2013).

29 Donato R. Intracellular and extracellular roles of S100 proteins. Microsc. Res. Tech. 60(6), 540-551 (2003).

30 Wright NT, Varney KM, Ellis KC et al. The three-dimensional solution structure of $\mathrm{Ca}(2+)$-bound S100A1 as determined by NMR spectroscopy. J. Mol. Biol. 353(2), 410-426 (2005).

31 Zimmer DB, Wright Sadosky P, Weber DJ. Molecular mechanisms of S100-target protein interactions. Microsc. Res. Tech. 60(6), 552-559 (2003).

32 Osterloh D, Ivanenkov VV, Gerke V. Hydrophobic residues in the $\mathrm{C}$-terminal region of S100A1 are essential for target protein binding but not for dimerization. Cell Calcium 24(2), 137-151 (1998).

33 Goch G, Vdovenko S, Kozlowska H, Bierzynski A. Affinity of S100A1 protein for calcium increases dramatically upon glutathionylation. FEBS J. 272(10), 2557-2565 (2005).

34 Zhukov I, Ejchart A, Bierzynski A. Structural and motional changes induced in apo-S100A1 protein by the disulfide formation between its Cys 85 residue and beta-mercaptoethanol. Biochemistry 47(2), 640-650 (2008).

35 Zhukova L, Zhukov I, Bal W, WyslouchCieszynska A. Redox modifications of the $\mathrm{C}$-terminal cysteine residue cause structural changes in S100A1 and S100B proteins. Biochim. Biophys. Acta 1742(1-3), 191-201 (2004).
36 Nowakowski M, Ruszczynska-Bartnik K, Budzinska $\mathrm{M}$ et al. Impact of calcium binding and thionylation of $S 100 \mathrm{~A} 1$ protein on its nuclear magnetic resonance-derived structure and backbone dynamics. Biochemistry 52(7), 1149-1159 (2013).

37 Lenarcic Zivkovic M, Zareba-Koziol M, Zhukova L, Poznanski J, Zhukov I, Wyslouch-Cieszynska A. Post-translational $\mathrm{S}$-nitrosylation is an endogenous factor fine tuning the properties of human S100A1 protein. J. Biol. Chem. 287(48), 40457-40470 (2012).

38 Hare JM, Stamler JS. NO/redox disequilibrium in the failing heart and cardiovascular system. J. Clin. Invest. 115(3), 509-517 (2005).

39 Most P, Boerries M, Eicher C et al. Distinct subcellular location of the $\mathrm{Ca}^{2+}$-binding protein S100A1 differentially modulates $\mathrm{Ca}^{2+}$-cycling in ventricular rat cardiomyocytes. J. Cell. Sci. 118(Pt 2), 421-431 (2005).

40 Gutierrez-Cruz G, Van Heerden AH, Wang K. Modular motif, structural folds and affinity profiles of the PEVK segment of human fetal skeletal muscle titin. J. Biol. Chem. 276(10), 7442-7449 (2001).

41 Heierhorst J, Kobe B, Feil SC et al. $\mathrm{Ca}^{2+} / \mathrm{S} 100$ regulation of giant protein kinases. Nature 380(6575), 636-639 (1996).

42 Maco B, Mandinova A, Durrenberger MB, Schafer BW, Uhrik B, Heizmann CW. Ultrastructural distribution of the S100A1 $\mathrm{Ca}^{2+}$-binding protein in the human heart. Physiol. Res. 50(6), 567-574 (2001).

43 Yamasaki R, Berri M, Wu Y et al. Titin-actin interaction in mouse myocardium: passive tension modulation and its regulation by calcium/S100A1. Biophys. J. 81(4), 2297-2313 (2001).

44 Boerries M, Most P, Gledhill JR et al. $\mathrm{Ca}^{2+}$-dependent interaction of S100A1 with F1-ATPase leads to an increased ATP content in cardiomyocytes. Mol. Cell. Biol. 27(12), 4365-4373 (2007).

- First study revealing S100A1 interaction with mitochondrial F1-ATPase in cardiomyocytes.

45 Brezova A, Heizmann CW, Uhrik B. Immunocytochemical localization of S100A1 in mitochondria on cryosections of the rat heart. Gen. Physiol. Biophys. 26(2), 143-149 (2007).

46 Kettlewell S, Most P, Currie S, Koch WJ, Smith GL. S100A1 increases the gain of excitation-contraction coupling in isolated 
rabbit ventricular cardiomyocytes. J. Mol. Cell. Cardiol. 39(6), 900-910 (2005).

47 Most P, Bernotat J, Ehlermann P et al. S100A1: a regulator of myocardial contractility. Proc. Natl Acad. Sci. USA 98(24), 13889-13894 (2001).

Most P, Remppis A, Pleger ST et al. Transgenic overexpression of the $\mathrm{Ca}^{2+}$ binding protein $S 100 \mathrm{~A} 1$ in the heart leads to increased in vivo myocardial contractile performance. J. Biol. Chem. 278(36), 33809-33817 (2003).

Volkers M, Loughrey CM, Macquaide N et al. S100A1 decreases calcium spark frequency and alters their spatial characteristics in permeabilized adult ventricular cardiomyocytes. Cell Calcium 41(2), 135-143 (2007).

50 Pleger ST, Shan C, Ksienzyk J et al. Cardiac AAV9-S100A1 gene therapy rescues post-ischemic heart failure in a preclinical large animal model. Sci. Transl. Med. 3(92), 92ra64 (2011).

- First study showing long-term therapeutic effectiveness and safety of adeno-associated virus (AAV)-S100A1 gene therapy in a large heart failure model.
51 Weber C, Neacsu I, Krautz B et al. Therapeutic safety of high myocardial expression levels of the molecular inotrope S100A1 in a preclinical heart failure model. Gene Ther. 21(2), 131-138 (2014).

52 Neubauer S, Horn M, Cramer M et al. Myocardial phosphocreatine-to-ATP ratio is a predictor of mortality in patients with dilated cardiomyopathy. Circulation 96(7), 2190-2196 (1997).

53 Fukushima H, Chung CS, Granzier H. Titin-isoform dependence of titin-actin interaction and its regulation by $\mathrm{S} 100 \mathrm{~A} 1 / \mathrm{Ca}^{2+}$ in skinned myocardium. J. Biomed. Biotechnol. 2010, 727239 (2010).

54 Pleger ST, Boucher M, Most P, Koch WJ. Targeting myocardial beta-adrenergic receptor signaling and calcium cycling for heart failure gene therapy. J. Card. Fail. 13(5), 401-414 (2007).

55 Usui A, Kato K, Abe T, Murase M, Tanaka M, Takeuchi E. S-100ao protein in blood and urine during open-heart surgery. Clin. Chem. 35(9), 1942-1944 (1989).

56 Usui A, Kato K, Sasa H et al. S-100ao protein in serum during acute myocardial infarction. Clin. Chem. 36(4), 639-641 (1990).
57 Matzinger P. The danger model: a renewed sense of self. Science 296(5566), 301-305 (2002).

58 Oppenheim JJ, Yang D. Alarmins: chemotactic activators of immune responses. Curr. Opin. Immunol. 17(4), 359-365 (2005).

59 Bianchi ME. DAMPs, PAMPs and alarmins: all we need to know about danger. J. Leukoc. Biol. 81(1), 1-5 (2007).

60 Foell D, Wittkowski H, Vogl T, Roth J. S100 proteins expressed in phagocytes: a novel group of damage-associated molecular pattern molecules. J. Leukoc. Biol. 81(1), 28-37 (2007).

61 Andrassy M, Volz HC, Igwe JC et al. High-mobility group box-1 in ischemiareperfusion injury of the heart. Circulation 117(25), 3216-26 (2008).

62 Pleger ST, Brinks H, Ritterhoff J et al. Heart failure gene therapy: the path to clinical practice. Circ. Res. 113(6), 792-809 (2013).

63 Ritterhoff J, Most P. Targeting S100A1 in heart failure. Gene Ther. 19(6), 613-621 (2012).

64 Rysä J, Leskinen H, Ilves M et al. Distinct upregulation of extracellular matrix genes in transition from hypertrophy to hypertensive heart failure. Hypertension 45(5), 927-933 (2005). 